PROCEEDINGS OF THE

AMERICAN MATHEMATICAL SOCIETY

Volume 139, Number 8, August 2011, Pages 2747-2751

S 0002-9939(2011)10699-3

Article electronically published on March 24, 2011

\title{
STABLY WEAKLY SHADOWING TRANSITIVE SETS AND DOMINATED SPLITTINGS
}

\author{
DAWEI YANG
}

(Communicated by Yingfei Yi)

\begin{abstract}
We prove that for any $C^{1}$-stably weakly shadowing transitive set $\Lambda$, either $\Lambda$ is a sink or a source or $\Lambda$ admits a dominated splitting.
\end{abstract}

\section{INTRODUCTION}

Shadowing properties have physical meaning: even if small errors occur at each iteration, one can track the resulting pseudo-orbit by a true orbit for a stable (hyperbolic) system. A generalization of the classical shadowing called weakly shadowing was introduced by [5]. The weakly shadowing property is $C^{0}$ and $C^{1}$ generic by [5, 6. One of the problems is to characterize the stably weakly shadowing diffeomorphisms. The following conjecture is given by S. Gan:

Conjecture. A diffeomorphism $f$ is $C^{1}$-stably weakly shadowing if and only if $f$ is tame.

Let's be more precise. Let $M$ be a compact $C^{\infty}$ Riemannian manifold without boundary. Let $\operatorname{Diff}^{1}(M)$ be the space of $C^{1}$ diffeomorphisms of $M$; by "space" we mean there is a usual $C^{1}$ metric defined on $\operatorname{Diff}^{1}(M)$. Assume $f \in \operatorname{Diff}^{1}(M)$. For any $x \in M, \operatorname{Orb}_{f}(x)=\left\{f^{n}(x)\right\}_{n \in \mathbb{Z}}$ is the orbit of $x$ with respect to $f$. For $\varepsilon>0,\left\{x_{n}\right\}_{n \in \mathbb{Z}}$ is called an $\varepsilon$-pseudo-orbit if $d\left(f\left(x_{n}\right), x_{n+1}\right)<\varepsilon$ for any $n \in \mathbb{Z}$. One says that $f$ has the weakly shadowing property if for any $\varepsilon>0$, there is $\delta>0$ such that for any $\delta$-pseudo-orbit $\left\{x_{n}\right\}_{n \in \mathbb{Z}}$, there exists $x \in M$ such that $\left\{x_{n}\right\}_{n \in \mathbb{Z}} \subset B(\operatorname{Orb}(x), \varepsilon)$, where $\operatorname{Orb}(x)$ is the orbit of $x$. Here $f$ is called $C^{1}$-stably weakly shadowing if there is a neighborhood $\mathcal{U}$ of $f$ such that any $g \in \mathcal{U}$ is weakly shadowing. Moreover $f$ is tame if there is a neighborhood $\mathcal{U}$ of $f$ such that any $g \in \mathcal{U}$ has only finitely many chain recurrent classes 1 Gan's conjecture is true if $\operatorname{dim} M=2$ 13. There is no answer on Gan's conjecture for higher dimensional diffeomorphisms.

If we focus on the "local" case, we can show that every $C^{1}$-stably weakly shadowing transitive set admits a dominated splitting. Let $\Lambda$ be a compact invariant set of $f$. We say that $\Lambda$ has the weakly shadowing property if for any $\varepsilon>0$, there is $\delta>0$ such that for any $\delta$-pseudo-orbit $\left\{x_{n}\right\}_{n \in \mathbb{Z}} \subset \Lambda$, there exists $x \in M$ such that $\left\{x_{n}\right\}_{n \in \mathbb{Z}} \subset B(\operatorname{Orb}(x), \varepsilon)$. For any set $U$, define $M_{U}(f)=\bigcap_{n \in \mathbb{Z}} f^{n}(U)$ to be the

Received by the editors March 10, 2010 and, in revised form, July 12, 2010.

2010 Mathematics Subject Classification. Primary 37-XX; Secondary 37C05, 37D30.

${ }^{1}$ This definition is given by S. Gan. Other definitions of "tame" are given by C. Bonatti and F. Abdenur [1].

(C)2011 American Mathematical Society 2747 
maximal invariant set of $f$ in $U$. For a compact invariant set $\Lambda$, we say that $\Lambda$ has the $C^{1}$-stably weakly shadowing property if there is a compact neighborhood $U$ of $\Lambda$ and a $C^{1}$ neighborhood $\mathcal{U}$ of $f$ such that $M_{U}(g)$ has the weakly shadowing property for any $g \in \mathcal{U}$. For an invariant set $\Lambda$, if there is an invariant splitting of the tangent bundle $T_{\Lambda} M=E \oplus F$, together with two constants $C>0$ and $\lambda \in(0,1)$ such that $\left\|\left.D f^{n}\right|_{E(x)}\right\|\left\|\left.D f^{-n}\right|_{F\left(f^{n}(x)\right)}\right\| \leq C \lambda^{n}$ for any $n \in \mathbb{N}$ and $x \in \Lambda$, one says that $\Lambda$ has a $(C, \lambda)$-dominated splitting; $\operatorname{dim} E$ is called the index of this dominated splitting. The notion of dominated splitting is much weaker than the notion of hyperbolic splitting. But dominated splitting is also a robust property and it is an important mechanism for many dynamical phenomena. Recall that a compact invariant set $\Lambda$ is called transitive if $\omega(x)=\Lambda$ for some $x \in \Lambda$.

Theorem A. For a transitive set $\Lambda$, if $\Lambda$ has the $C^{1}$-stably weakly shadowing property and if $\Lambda$ is neither a hyperbolic sink nor a hyperbolic source, then $\Lambda$ admit a dominated splitting.

One should notice that [7] proved a similar result for homoclinic classes. Each homoclinic class is transitive from hyperbolic theory.

\section{The REDUCTION OF THE PROBLEM AND PERIODIC LINEAR COCYCLES}

For a periodic point $p$ of $f \in \operatorname{Diff}^{1}(M)$, we list all the eigenvalues of $D f^{\pi(p)}$ as $\left\{\lambda_{1}, \lambda_{2}, \cdots, \lambda_{d}\right\}$ which verifies

$$
\left|\lambda_{1}\right| \leq\left|\lambda_{2}\right| \leq \cdots \leq\left|\lambda_{d}\right|
$$

One says that $p$ is an almost source if $\left|\lambda_{1}\right|=1 ; p$ is an almost sink if $\left|\lambda_{d}\right|=1$. The following lemma is from [7, Lemma 3.2]:

Lemma 2.1. If $\Lambda$ is a $C^{1}$-stably weakly shadowing set of $f$, then there is a neighborhood $\mathcal{U}$ of $f$ and a neighborhood $U$ of $\Lambda$ such that $M_{U}(g)$ contains neither almost sinks nor almost sources for any $g \in \mathcal{U}$.

In this paper, the metric between compact sets is the Hausdorff metric. We use $d_{H}$ to denote the distance of the Hausdorff distance. The limits of compact sets are under the Hausdorff distance. The following lemma concerns the limit of uniformly dominated splitting. One can see a proof in [3, Lemma 1.4].

Lemma 2.2. Assume $C>0$ and $\lambda \in(0,1)$. If there are a sequence of diffeomorphisms $\left\{f_{n}\right\}$ and a sequence of compact sets $\left\{\Lambda_{n}\right\}$ such that $\Lambda_{n}$ is a compact invariant set of $f_{n}$ and $\Lambda_{n}$ admits a $(C, \lambda)$-dominated splitting of index $i$ with respect to $f_{n}$, and then if $\Lambda=\lim _{n \rightarrow \infty} \Lambda_{n}$ exists, then $\Lambda$ admits a $(C, \lambda)$-dominated splitting of index $i$ with respect to $f$.

Let $p_{n}$ be a periodic point of $f_{n}$ with period $\pi\left(p_{n}\right)$. One says that $\left\{p_{n}\right\}$ is uniformly contracting at the period if there are $C>0, \lambda \in(0,1)$ and $\iota \in \mathbb{N}$ such that for any $p_{n}$ with $\pi\left(p_{n}\right)>\iota$, then

$$
\prod_{i=0}^{\left[\pi\left(p_{n}\right) / \iota\right]-1}\left\|D f_{n}^{\iota}\left(f_{n}^{i \iota}\left(p_{n}\right)\right)\right\| \leq C \lambda^{\left[\pi\left(p_{n}\right) / \iota\right]} .
$$

One says that $\left\{p_{n}\right\}$ is uniformly expanding at the period if it is uniformly contracting at the period for $\left\{f_{n}^{-1}\right\}$.

One can extract the following lemma from [11] and [10, Lemma II.4, Lemma II.5]. 
Lemma 2.3. Assume $\lim _{n \rightarrow \infty} f_{n}=f$. If $p_{n}$ is a periodic sink of $\left\{f_{n}\right\}$, then either $\left\{p_{n}\right\}$ is uniformly contracting at the period or there is a sequence of diffeomorphisms $\left\{g_{n}\right\}$ such that $\operatorname{Orb}_{f_{n}}\left(p_{n}\right)$ is an almost sink of $g_{n}$ and $\lim _{n \rightarrow \infty} g_{n}=f$.

Similarly, one can get similar results for sources.

Lemma 2.4. Let $\Lambda$ be a transitive set of $f$. If there is a sequence of diffeomorphisms $\left\{f_{n}\right\}$ such that

- $f_{n}$ has a periodic point $p_{n}$ and $\left\{p_{n}\right\}$ is uniformly contracting at the period,

- $\lim _{n \rightarrow \infty} \operatorname{Orb}_{f_{n}}\left(p_{n}\right)$ exists and it is a subset of $\Lambda$,

then $\Lambda$ is a sink.

One can get similar results for $f^{-1}$ in the same manner.

Proof. One can see the proof of this lemma in [8, Lemma 3.2-Lemma 3.4].

Proposition 2.5. Assume that $\Lambda$ is a $C^{1}$-stably weakly shadowing set. Also assume that $\Lambda$ is transitive and $\Lambda$ is not a periodic orbit. Then there is a neighborhood $\mathcal{U}$ of $f$ and a neighborhood $U$ of $\Lambda$ such that any $g \in \mathcal{U}$ has neither sinks nor sources in $U$.

Proof. We will prove this proposition by reductio ad absurdum. If the conclusion is not true, without loss of generality one can assume that there is a sequence of diffeomorphisms $\left\{f_{n}\right\}$ such that each $f_{n}$ has a periodic sink $p_{n}$ such that $\lim _{n \rightarrow \infty} \operatorname{Orb}_{f_{n}}\left(p_{n}\right)$ exists and it is a subset of $\Lambda$. By Lemma 2.3, either $\left\{p_{n}\right\}$ is uniformly contracting at the period or there is a sequence of diffeomorphisms $\left\{g_{n}\right\}$ such that $\operatorname{Orb}_{f_{n}}\left(p_{n}\right)$ is an almost sink of $g_{n}$ and $\lim _{n \rightarrow \infty} g_{n}=f$. Since $\Lambda$ is a $C^{1}$-stably weakly shadowing set, by Lemma 2.1, $\left\{p_{n}\right\}$ is uniformly contracting at the period. Then by Lemma 2.4 $\Lambda$ is a periodic sink. This contradicts the fact that $\Lambda$ is not a periodic orbit.

Pugh 12 proved the following lemma, which guarantees the existence of periodic orbits by $C^{1}$ perturbations:

Lemma 2.6. For any $C^{1}$ neighborhood $\mathcal{U}$ of $f$ and for any non-periodic point $x$ with the property $x \in \omega(x)$, there are $N \in \mathbb{N}$ and $\varepsilon_{0}>0$ such that for any $\varepsilon \in\left(0, \varepsilon_{0}\right)$ and $n \geq N$, there is $g \in \mathcal{U}$ with the following properties:

- $g(z)=f(z)$ for any $z \in M \backslash\left(\bigcup_{i=0}^{n} B\left(f^{i}(x), \varepsilon\right)\right)$.

- There is a periodic point $y$ of $g$ such that $y \in B(x, \varepsilon)$ and $\operatorname{Orb}_{g}(y) \cap(M \backslash$ $\left.\left(\bigcup_{i=0}^{n} B\left(f^{i}(x), \varepsilon\right)\right)\right) \subset \operatorname{Orb}_{f}(x)$.

We will prove the following folklore result:

Lemma 2.7. Let $\Lambda$ be a transitive set of $f$. For any $\varepsilon>0$ and for any neighborhood $\mathcal{U}$ of $f$, there is a periodic point $p$ of $g \in \mathcal{U}$ such that $d_{H}\left(\operatorname{Orb}_{g}(p), \Lambda\right)<\varepsilon$.

Proof. Since $\Lambda$ is transitive, there exists $x \in \Lambda$ such that $\omega(x)=\Lambda$. For $\varepsilon>0$, there exists $N_{1} \in \mathbb{N}$ such that $d_{H}\left(\left\{x, f(x), \cdots, f^{n}(x)\right\}, \Lambda\right)<\varepsilon / 2$ for any $n>N_{1}$. For any neighborhood $\mathcal{U}$ of $f$, one can get two constants $N$ and $\varepsilon_{0}$ from Pugh's closing lemma (Lemma 2.6). Without loss of generality, one can assume that $N>N_{1}$ and $\varepsilon_{0}>\varepsilon$. For $n>N$, by Pugh's closing lemma, there are $g \in \mathcal{U}$ and $y \in B(x, \varepsilon / 2)$ such that

- $y$ is a periodic point of $g$;

- when $g^{i}(y) \in\left(M \backslash\left(\bigcup_{i=0}^{n} B\left(f^{i}(x), \varepsilon\right)\right)\right), g^{i}(y) \in B\left(f^{i}(x), \varepsilon / 2\right)$ and $g^{i}(y) \in$ $\operatorname{Orb}_{f}(x)$. 
From the above properties one can check that the conclusion of this lemma is true.

As a corollary,

Corollary 2.7.1. Let $\Lambda$ be a transitive set of $f$. There are a sequence of diffeomorphisms $\left\{f_{n}\right\}$ and a sequence of points $\left\{p_{n}\right\}$ such that $p_{n}$ is a periodic point of $f_{n}$ and $\lim f_{n}=f$ and $\lim \operatorname{Orb}\left(p_{n}\right)=\Lambda$.

Now we give the proof of the main theorem. Let $\Lambda$ be a $C^{1}$-stably weakly shadowing transitive set of $f$. Moreover, $\Lambda$ is neither a sink nor a source. We will prove the main theorem by contradiction; i.e., we assume that $\Lambda$ does not admit any dominated splitting.

Claim. Under the above assumptions, $\Lambda$ is not a periodic orbit.

Proof. If $\Lambda$ is a periodic orbit, then $\Lambda$ is neither an almost sink nor an almost source. So $\lambda_{d}>1$ and $\lambda_{1}<1$, and the periodic orbit admits a dominated splitting.

Since $\Lambda$ is transitive, by Corollary 2.7.1 there are a sequence of diffeomorphisms $\left\{f_{n}\right\}$ and a sequence of points $\left\{p_{n}\right\}$ such that $p_{n}$ is a periodic point of $f_{n}$ and $\lim f_{n}=f$ and $\lim \operatorname{Orb}\left(p_{n}\right)=\Lambda$. Since $\Lambda$ is not a periodic orbit, one has $\pi\left(p_{n}\right) \rightarrow \infty$ as $n \rightarrow \infty$.

Let $\Sigma=\coprod_{n \in \mathbb{N}}\left\{p_{n}, f\left(p_{n}\right), \cdots, f^{\pi\left(p_{n}\right)-1}\left(p_{n}\right)\right\}$. One can define a natural $d$ dimensional vector bundle $E$ on $\Sigma$ as following: for any $x \in \Sigma$, the fibre on $x$ is $T_{x} M$. For any $i \in\left[0, \pi\left(p_{n}\right)-1\right] \cap \mathbb{N}$, we define $h\left(f_{n}^{i}\left(p_{n}\right)\right)=f_{n}^{i+1}\left(p_{n}\right)$ and $\left.A\right|_{E\left(f_{n}^{i}\left(p_{n}\right)\right)}=D f_{n}\left(f_{n}^{i}\left(p_{n}\right)\right)$. Thus $\mathcal{A}=(\Sigma, h, E, A)$ is a bounded large periodic system as in [4, Section 2.1-Section 2.3]. Then by [4, Theorem 2.2],

- either there is an infinite subset $\Sigma^{\prime} \subset \Sigma$ which is invariant by $h$ such that the periodic linear cocycle $\mathcal{A}^{\prime}=\left(\Sigma^{\prime}, h,\left.E\right|_{\Sigma^{\prime}}, A\right)$ admits a dominated splitting, which means that there is an invariant splitting $\left.E\right|_{\Sigma^{\prime}}=E^{c s} \oplus E^{c u}$ with respect to $A$, together with two constants $C>0$ and $\lambda \in(0,1)$, such that for any $x \in \Sigma^{\prime}$, for any $n \in \mathbb{N}$, one has $\left\|\left.A^{n}\right|_{E^{c s}(x)}\right\|\left\|\left.A^{-n}\right|_{E^{c u}\left(h^{n}(x)\right)}\right\| \leq C \lambda^{n}$

- or there is a perturbation $\mathcal{B}$ of $\mathcal{A}$ and an infinite invariant subset $\Sigma^{\prime}$ of $\Sigma$ such that for any $x \in \Sigma^{\prime}$, all eigenvalues of $B\left(h^{\pi(x)-1}(x)\right) \circ B\left(h^{\pi(x)-1}(x)\right) \circ$ $\cdots \circ B(x)$ are real, with the same modulus.

With the help of Franks' Lemma 9, 10, we can translate the above statement for diffeomorphims: by taking a subsequence if necessary, either there are constants $C>0$ and $\lambda \in(0,1)$ such that there is a $(C, \lambda)$-dominated splitting on the orbit $\left\{p_{n}\right\}$ or there is a sequence of diffeomorphisms $\left\{g_{n}\right\}$ such that $\lim _{n \rightarrow \infty} g_{n}=f$ and $\operatorname{Orb}_{f_{n}}\left(p_{n}\right)$ is also a periodic orbit of $g_{n}$ and all eigenvalues of $D g_{n}^{\pi\left(p_{n}\right)}\left(p_{n}\right)$ are all real and with the same modulus.

For the first case, we can get that $\Lambda$ has a dominated splitting by Lemma 2.2. In the second case, one can get that $\Lambda$ is either a sink or a source, or by an arbitrarily small perturbation there is an almost sink or an almost source in an arbitrarily small neighborhood of $\Lambda$, which contradicts the fact that $\Lambda$ is $C^{1}$-stably weakly shadowing. 


\section{ACKNOWLEDGEMENTS}

The author would like to thank Jilin University for support in getting his scientific research started and for the support of the project "The spectral decomposition theorems of star vector fields".

\section{REFERENCES}

[1] F. Abdenur, Generic robustness of spectral decompositions, Ann. Sci. École Norm. Sup., 36(2003), 213-224. MR 1980311 (2004b:37032)

[2] C. Bonatti and S. Crovisier, Récurrence et généricité, Invent. Math., 158 (2004), 33-104. MR2090361 (2007b:37036)

[3] C. Bonatti, L. J. Díaz and E. R. Pujals, A $C^{1}$-generic dichotomy for diffeomorphisms: Weak forms of hyperbolicity or infinitely many sinks or sources, Annals of Math. (2), 158 (2003), 355-418. MR2018925 (2007k:37032)

[4] C. Bonatti, N. Gourmelon and T. Vivier, Perturbations of the derivative along periodic orbits, Ergodic Theory Dynam. Systems, 26(2006), 1307-1337. MR2266363(2007i:37062)

[5] R. M. Corless and S. Yu. Pilyugin, Approximate and real trajectories for generic dynamical systems, J. Math. Anal. Appl., 189(1995), 409-423. MR1312053 (95k:58090)

[6] S. Crovisier, Periodic orbits and chain transitive sets of $C^{1}$-diffeomorphisms, Publ. Math. Inst. Hautes Études Sci., 104(2006), 87-141. MR2264835 (2008f:37047)

[7] S. Gan, K. Sakai and L. Wen, $C^{1}$-stably weakly shadowing homoclinic classes admit dominated splittings, Disc. Cont. Dynam. Syst., 27(2008), 945-957. MR2600767

[8] S. Gan and L. Wen, Nonsingular star flows satisfy Axiom A and the no-cycle condition, Invent. Math., 164(2006), 279-315. MR2218778(2007j:37045)

[9] J. Franks, Necessary conditions for stabilty of diffeomorphisms, Trans. Amer. Math. Soc., 158(1971), 301-308. MR0283812(44:1042)

[10] R. Mañé, An ergodic closing lemma, Ann. Math. (2), 116(1982), 503-540. MR678479 (84f:58070)

[11] V. Pliss, A hypothesis due to Smale, Diff. Eq., 8(1972), 203-214.

[12] C. Pugh, The closing lemma, Amer. J. Math., 89(1967), 956-1009. MR0226669 (37:2256)

[13] K. Sakai, Diffeomorphisms with weak shadowing, Fund. Math., 168(2001), 57-75. MR1835482(2002d:37041)

School of Mathematics, Jilin University, Changchun, 130012, People's Republic of CHINA

E-mail address: yangdw1981@gmail.com 